\title{
Beyond Blowing: Oral \\ Appliance Therapy, Surgery, and Transcranial Magnetic Stimulation
}

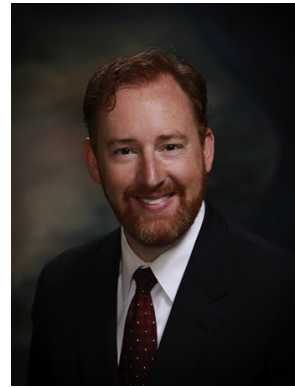

Jamison R. Spencer, DMD, MS

Editor

It has been my pleasure to act as editor of this issue of Sleep Medicine Clinics.

Not everyone can tolerate positive airway pressure (PAP) therapy. And PAP isn't always the best therapeutic option for the obstructive sleep apnea (OSA) patient.

Oral appliance therapy and surgical approaches in the treatment of OSA have been around for a very long time and have provided alternative, and sometimes adjunctive, options for our patients.

If you are a physician, you may have heard that oral appliance therapy is only for mild cases and causes TMJ problems, bite changes, and other problems. Physicians and dentists alike may have heard that surgery in general is ineffective and only bimaxillary advancement (also known as orthognathic surgery) works (other than tracheostomy, of course). In this issue, we address these areas of potential concern and controversy.

For physicians reading this, I hope it provides you with a good overview of options that are available to your OSA patients that you may be less familiar with, as well as providing a window into the world of those who dedicate their professional careers to providing these therapies and procedures. You'll learn that qualified dentists well trained in the care of patients with OSA go far beyond "just fitting a piece of plastic," and that the preparatory work going into skeletal surgery is as critical to success as the surgical procedure itself.

For dentists reading this, you will be able to learn everything from the history of oral appliance therapy to the latest techniques, procedures, and protocols from initial screening to final follow-up. This publication may be used as a helpful guide and reference in your evaluation and treatment of both adults and children as well as help you better understand the surgical approaches from which many of your patients may benefit.

To all who take the time to review this issue, I am confident that you will be rewarded with a deeper understanding of nonsurgical and surgical approaches that are available to help the patients you serve. As physicians, dentists, and other health care providers work together through the screening, diagnosis, and treatment process, our patients are the ones who will benefit from better, more predictable, and more effective outcomes.

Jamison R. Spencer, DMD, MS

The Center for Sleep Apnea and TMJ Redwood Dental Specialty Plaza 6287 South Redwood Road, Suite 101

Salt Lake City, UT 84123, USA

E-mail address: Jamison@JamisonSpencer.com 